\title{
A note on Brouwer's weak continuity principle and the transfer principle in nonstandard analysis
}

\author{
ERIK PALMGREN ${ }^{1}$
}

\begin{abstract}
A well-known model of nonstandard analysis is obtained by extending the structure of real numbers using an ultrapower construction. A constructive approach due to Schmieden and Laugwitz uses instead a reduced power construction modulo a cofinite filter, but has the drawback that the transfer principle is weak. In this paper it is shown that this principle can be strengthened by employing Brouwerian continuity axioms familiar from intuitionistic systems. We end by commenting on the relation between the transfer principle and Ishihara's boundedness principle.
\end{abstract}

2000 Mathematics Subject Classification 03F55, 03H05, 26E35 (primary); $03 \mathrm{~F} 60$ (secondary)

Keywords: intuitionistic mathematics, nonstandard analysis, transfer principle

\section{Introduction}

A well-known approach to nonstandard analysis is obtained by extending the structure of real numbers using an ultrapower construction (see [4]). There is also a constructive approach, originating with Schmieden and Laugwitz [16], which uses a reduced power construction modulo a cofinite filter. It has been investigated and developed by MartinLöf [9], the author [12,13] and Schuster [17] in the context of BISH, constructive mathematics in the sense of Bishop [1]. A drawback of this approach is that the transfer principle is rather weak. We show here that this principle can be strengthened by assuming Brouwerian continuity axioms familiar from intuitionistic systems.

The Schmieden-Laugwitz extension can be described in elementary model-theoretic terms as follows. Let $M$ be a first-order structure with respect to the many-sorted signature $L$. All sorts are supposed to be inhabited. Construct $M^{\star}$, a first-order

\footnotetext{
${ }^{1}$ Supported by grants from the Swedish Research Council (VR) and the EU Marie Curie IRSES project CONSTRUMATH.
} 
structure, whose underlying sets are of the form $S^{\mathbb{N}}$, the set of sequences of elements in $S$ for each sort $S$ in $M$. Two sequences $f$ and $g$ are defined to be equal in the structure as follows $f=S_{S^{\star}} g \Longleftrightarrow_{\text {def }}(\exists k)(\forall i \geq k) f(i)=g(i)$. More generally we define for each relation $R$ in the signature $L$ :

$$
R^{\star}\left(f_{1}, \ldots, f_{n}\right) \Longleftrightarrow{ }_{\text {def }}(\exists k)(\forall i \geq k) R\left(f_{1}(i), \ldots, f_{n}(i)\right)
$$

for $f_{1}, \ldots, f_{n} \in M^{\star}$. For each function $g$ in the signature $L$ define

$$
g^{\star}\left(f_{1}, \ldots, f_{n}\right)=\left(i \mapsto g\left(f_{1}(i), \ldots, f_{n}(i)\right)\right) .
$$

The below transfer theorem between the structures $M$ and $M^{\star}$ can be proved by induction on formulas, using countable choice for the existential case. A first order formula is called existential conjunctive if it is built up from atomic formulas using only $\wedge$ and $\exists$. The proof is essentially contained in Martin-Löf [9], but see also [12].

Theorem 1.1 For each $L$-structure $M$, for each existential conjunctive formula $\varphi\left(x_{1}, \ldots, x_{n}\right)$ in the signature $L$, and for parameters $f_{1} \in S_{1}^{\star}, \ldots, f_{n} \in S_{n}^{\star}$ :

$$
M^{\star} \models \varphi\left(f_{1}, \ldots, f_{n}\right) \Longleftrightarrow(\exists k)(\forall i \geq k) M \models \varphi\left(f_{1}(i), \ldots, f_{n}(i)\right) .
$$

Below we consider general quantified conjuctive formulas. A first order formula is called quantified conjunctive if it is built up from atomic formulas using only $\wedge, \exists$ and $\forall$. Such a formula may easily be seen to be equivalent to a prenex formula of the form

$$
\left(Q_{1} y_{1} \in T_{1}\right) \cdots\left(Q_{m} y_{m} \in T_{m}\right) \psi\left(y_{1}, \ldots, y_{m}, x_{1}, \ldots, x_{n}\right)
$$

where $\psi$ is a conjunction of atomic formulas and each $Q_{i}$ is either $\exists$ and $\forall$.

Remark It is possible to obtain a full transfer principle constructively if the model notion is relaxed, by using sheaf semantics [10] or using non-standard truth [9]. However, with standard semantics, full transfer is impossible [13, p. 234].

\section{Extended transfer principles}

We shall use a Brouwerian type axiom to extend the transfer principle of Theorem 1.1. The weak continuity principle for a set $S$ says that for any predicate $P \subseteq\left(S^{\mathbb{N}}\right) \times \mathbb{N}$

$$
(\forall \alpha)(\exists n) P(\alpha, n) \Longrightarrow(\forall \alpha)(\exists n)(\exists k)(\forall \beta)[(\forall i<k) \beta(i)=\alpha(i) \Rightarrow P(\beta, n)] .
$$

Here $\alpha$ and $\beta$ varies over $S^{\mathbb{N}}$. 
Remark The special case when $S=\mathbb{N}$ is the familiar WC-N ([18]). We do not know of a model for the general case but the axiom scheme is certainly possible to justify similarly to the special case. Moerdijk and van der Hoeven [3] gives model for WC-N which might be a candidate for generalization.

Under the assumption of the above axiom we can strengthen Theorem 1.1.

Theorem 2.1 Assume that weak continuity principle holds for each sort of the $L$ structure $M$. For each quantified conjunctive formula $\varphi\left(x_{1}, \ldots, x_{n}\right)$ in the signature $L$ and for parameters $f_{1} \in S_{1}^{\star}, \ldots, f_{n} \in S_{n}^{\star}$ :

$$
M^{\star} \models \varphi\left(f_{1}, \ldots, f_{n}\right) \Longleftrightarrow(\exists k)(\forall i \geq k) M \models \varphi\left(f_{1}(i), \ldots, f_{n}(i)\right) .
$$

Proof. In view of the inductive proof of Theorem 1.1 we need only check the $\forall$-case.

$(\Leftarrow)$ Suppose that $(\exists k)(\forall i \geq k) M=(\forall y \in S) \varphi\left(f_{1}(i), \ldots, f_{n}(i), y\right)$, that is $(\exists k)(\forall i \geq$ $k)(\forall y \in S) M \models \varphi\left(f_{1}(i), \ldots, f_{n}(i), y\right)$. Hence for any $g \in S^{\star}$,

$$
(\exists k)(\forall i \geq k) M=\varphi\left(f_{1}(i), \ldots, f_{n}(i), g(i)\right) .
$$

The inductive hypothesis gives $M^{\star} \models(\forall y \in S) \varphi\left(f_{1}(i), \ldots, f_{n}(i), y\right)$.

$(\Rightarrow)$ Suppose $M^{\star} \models(\forall y \in S) \varphi\left(f_{1}(i), \ldots, f_{n}(i), y\right)$. This implies by the inductive hypothesis that for each $g: \mathbb{N} \rightarrow S$ there exists $k \in \mathbb{N}$ such that

$$
(\forall i \geq k) M=\varphi\left(f_{1}(i), \ldots, f_{n}(i), g(i)\right) .
$$

Hence by the weak continuity principle for $S$, there is $\ell \in \mathbb{N}$ so that for each $h: \mathbb{N} \rightarrow S$ with $(\forall j<\ell) h(j)=g(j)$ it holds that

$$
(\forall i \geq k) M=\varphi\left(f_{1}(i), \ldots, f_{n}(i), h(i)\right) .
$$

Let $g(i)=m$ for all $i$, where $m$ is a fixed element in $S$. Then we get $k$ and $\ell$ so that for each $h: \mathbb{N} \rightarrow S$ with $(\forall j<\ell) h(j)=g(j)$ it holds that

$$
(\forall i \geq k) M=\varphi\left(f_{1}(i), \ldots, f_{n}(i), h(i)\right) .
$$

We may assume that $k \geq \ell$. Let $y$ be an arbitrary element in $S$. Define $h: \mathbb{N} \rightarrow S$ by letting $h(i)=g(i)$ for $i<\ell$ and $h(i)=y$ for $i \geq \ell$. Then since $k \geq \ell$, the statement (2) gives

$$
(\forall i \geq k) M \models \varphi\left(f_{1}(i), \ldots, f_{n}(i), y\right) .
$$

But $y$ was arbitrary, so

$$
(\forall i \geq k) M \models(\forall y \in S) \varphi\left(f_{1}(i), \ldots, f_{n}(i), y\right) .
$$


This proves the equivalence.

We note that the direction $(\Leftarrow)$ did not use the continuity principle. Indeed this direction can be further strengthened to apply to so-called constructive Horn formulas along the lines of [12]. Similarly, the saturation principles that depend on transfer principles can be improved. We will not pursue this is in the present paper.

A special case of the theorem is worth noting:

Corollary 2.2 Assuming WC-N, the equivalence (1) holds for $M=\mathbb{N}$, the structure of natural numbers, with all functions and relations.

A stronger classical theorem is due to Palyutin [14]. A formula is called a Palyutin formula if it is built up inductively from atomic formulas using $\forall, \exists, \wedge$ and the construction $(\exists x \varphi) \wedge(\forall x)(\varphi \rightarrow \psi)$ where $\varphi$ and $\psi$ are Palyutin formula.

Theorem 2.3 (Palyutin) Assume PEM and AC. Then Theorem 2.1 holds for all Palyutin formulas $\varphi$.

\section{Transfer and boundedness}

A subset $A \subseteq \mathbb{N}$ is bounded if there is $n \in \mathbb{N}$ so that $A \subseteq\{0,1,2, \ldots, n\}$. A useful characterization $[6,15]$ of a pseudo-bounded subset $A \subseteq \mathbb{N}$ is that for each $f: \mathbb{N} \rightarrow A$ there is some $k$ such that $f(n) \leq n$ for all $n \geq k$. The boundedness principle BD of Ishihara [5] is

Every inhabited pseudo-bounded set of natural numbers, is bounded.

A weaker principle $\mathrm{BD}-\mathrm{N}$ is:

Every inhabited countable pseudo-bounded set of natural numbers, is bounded.

The principle BD-N has the intriguing property of being a theorem of classical mathematics CLASS, intuitionistic mathematics INT and Russian recursive mathematics RUSS (using the nomenclature of [2]), and yet it is not provable in BISH as shown by Lietz [8]. BD-N is equivalent to the completeness of the uniform space $\mathcal{D}(\mathbf{R})$ of test functions in distribution theory [6] and to several important properties involving sequential continuity [5]. 
For each $S$ and $g: S \rightarrow \mathbb{N}$ let $\left(\operatorname{Tr}_{g}\right)$ be the proposition

$$
\left(\forall x \in S^{\star}\right) g^{\star}(x) \leq^{\star} \eta \Longleftrightarrow(\exists k)(\forall i \geq k)(\forall x \in S) g(x) \leq \eta(i),
$$

where $\eta: \mathbb{N} \rightarrow \mathbb{N}$ is the identity $\eta(i)=i$. Note that $\left(\operatorname{Tr}_{g}\right)$ is an instance of Theorem 2.1. Parts $(\Leftarrow)$ of the following characterization are due to Nordvall-Forsberg [11]. It can be regarded as a nonstandard formulation of Richman's criterion [15], and suggests that the boundedness principles may have further interesting strengthenings.

Theorem $3.1 \quad$ (a) BD holds if, and only if, for every inhabited set $S$ and each $g: S \rightarrow \mathbb{N},\left(\operatorname{Tr}_{g}\right)$ holds.

(b) BD-N holds if, and only if, for each $g: \mathbb{N} \rightarrow \mathbb{N}$, $\left(\operatorname{Tr}_{g}\right)$ holds.

Proof. (a, $\Leftarrow$ ) Suppose that $A \subseteq \mathbb{N}$ is an inhabited pseudo-bounded set. According to Richman's characterization this means that for each sequence $x: \mathbb{N} \rightarrow A$, there is $k \in \mathbb{N}$ so that $x(n) \leq n$ for all $n \geq k$. Letting $g: A \hookrightarrow \mathbb{N}$ be the inclusion, we thus have

$$
\left(\forall x \in A^{\star}\right) g^{\star}(x) \leq^{\star} \eta
$$

where $\eta(n)=n$. Hence by $\left(\operatorname{Tr}_{g}\right)(\exists k)(\forall n \geq k)(\forall x \in A) g(x) \leq \eta(n)$. Letting $n=k$ we see that $A$ is bounded by $k$.

$(\mathrm{a}, \Rightarrow)$ Assume BD. Let $g: S \rightarrow \mathbb{N}$ be arbitrary with $S$ inhabited. Note that the direction $(\Leftarrow)$ of $\left(\operatorname{Tr}_{g}\right)$ is trivial and does not require BD. We prove the opposite direction. Suppose

$$
\left(\forall x \in S^{\star}\right) g^{\star}(x) \leq^{\star} \eta .
$$

Thus for every $x: \mathbb{N} \rightarrow S$, there is $k$ such that $g(x(n)) \leq n$ for $n \geq k$. Let $A=\operatorname{Im}(g)$. For any $f: \mathbb{N} \rightarrow A$, there is by countable choice $x: \mathbb{N} \rightarrow S$ so that $g(x(n))=f(n)$ for all $n \in \mathbb{N}$. Hence $f(n) \leq n$ for all $n \geq k$. Thus $A$ is inhabited and pseudobounded, so by BD the set $A$ is bounded by some $N$. Hence $(\forall x \in S) g(x) \leq N$. Thus $(\forall n \geq N)(\forall x \in A) g(x) \leq \eta(n)$ as required.

(b, $\Leftarrow$ ) Observe that if $A$ is inhabited and countable, then $A=\operatorname{Im}(g)$ for some $g: \mathbb{N} \rightarrow \mathbb{N}$. Suppose $A$ is pseudo-bounded. Let $x: \mathbb{N} \rightarrow \mathbb{N}$ be arbitrary then $f(n)=g(x(n)) \in A$ for all $n \in \mathbb{N}$. Thus there is $k$ with $f(n) \leq n$ for all $n \geq k$. Hence $\left(\forall x \in \mathbb{N}^{\star}\right) g^{\star}(x) \leq^{\star} \eta$ and thus $\left(\operatorname{Tr}_{g}\right)$ implies $(\exists k)(\forall n \geq k)(\forall x \in \mathbb{N}) g(x) \leq \eta(n)$. Hence $A$ is bounded by $k$, thus proving BD-N.

$(\mathrm{b}, \Rightarrow)$ We note that for the case $S=\mathbb{N}$ in $(\mathrm{a}, \Rightarrow)$ BD-N suffices. 
Remark The principle BD-N is provable [5] using Markov's principle MP and a version of Church Thesis CPF. We do not know whether any significant instance of the transfer Theorem 2.1, apart from what is given by the above characterization, is provable using these axioms.

A generalization of boundedness relative to a relation is suggested by the above. Let $R \subseteq X \times \mathbb{N}$ be any binary relation. Write $R_{j}=\{x \in X:(x, j) \in R\}$. A subset $M \subseteq X$ is $R$-bounded if for some $k \in \mathbb{N}$

$$
M \subseteq \bigcap_{j \geq k} R_{j}
$$

The subset $M$ is $R$-pseudo-bounded, if for any sequence $f: \mathbb{N} \rightarrow M$, there is $k$ so that for all $j \geq k$

$$
f(j) \in R_{j}
$$

Clearly for $X=\mathbb{N}$ and $R=\leq$ these notions coincide with boundedness and pseudoboundedness respectively.

For $R \subseteq X \times \mathbb{N}$ the $\mathrm{BD}_{R}$ principle is: every inhabited $R$-pseudo-bounded subset of $X$ is $R$-bounded.

The proof of the following characterization is analogous to that of Theorem 3.1 (a) above.

Proposition 3.2 For $R \subseteq X \times \mathbb{N}$ the principle $\mathrm{BD}_{R}$ is equivalent to the following transfer statement: for every inhabited $S$ and every $g: S \rightarrow X$,

$$
\left(\forall x \in S^{\star}\right) R^{\star}\left(g^{\star}(x), \eta\right) \Longleftrightarrow(\exists k)(\forall i \geq k)(\forall x \in S) R(g(x), \eta(i))
$$

where $\eta(i)=i$.

\section{References}

[1] E. Bishop, Foundations of Constructive Analysis, McGraw-Hill 1967.

[2] D.S. Bridges and F. Richman, Varieties of constructive mathematics, Cambridge University Press 1987.

[3] G. van der Hoeven and I. Moerdijk, Sheaf models for choice sequences, Annals of Pure and Applied Logic 27 (1984), 63-107; doi: 10.1016/0168-0072(84)90035-6.

[4] A.E. Hurd and P.A. Loeb, An introduction to nonstandard real analysis, Academic Press 1985. 
[5] H. Ishihara. Continuity Properties in Constructive Mathematics, Journal of Symbolic Logic 57(1992), 557-565.

[6] H. Ishihara and S. Yoshida, A constructive look at the completeness of the space $\mathcal{D}(\mathbf{R})$, Journal of Symbolic Logic 67 (2002), 1511 - 1519.

[7] D. Laugwitz, Curt Schmieden's approach to infinitesimals: An eye-opener to the historiography of anlaysis, in: P. Schuster, U. Berger and H. Osswald (eds.), Reuniting the Antipodes - Constructive and Nonstandard Views of the Continuum, Kluwer 2001.

[8] P. Lietz, From Constructive Mathematics to Computable Analysis via the Realizability Interpretation, Doctoral dissertation, TU Darmstadt, 2004.

[9] P. Martin-Löf, Mathematics of infinity, in: P. Martin-Löf and G. Mints (eds.), COLOG88, International Conference on Computer Logic Tallinn, USSR, December 1216, 1988 Proceedings, Lecture Notes in Computer Science, vol. 417, 146-197, Springer 1990; doi: 10.1007/3-540-52335-9_54.

[10] I. Moerdijk, A model for intuitionistic nonstandard arithmetic, Annals of Pure and Applied Logic 73(1995), 37 - 51; doi: 10.1016/0168-0072(93)E0071-U.

[11] F. Nordvall Forsberg, Constructive aspects of models for non-standard analysis, MSc Thesis, Uppsala University, Department of Mathematics Project Report 2009:10.

[12] E. Palmgren, A constructive approach to nonstandard analysis, Annals of Pure and Applied Logic 73(1995), 297-325; doi: 10.1016/0168-0072(94)00030-7.

[13] E. Palmgren, Developments in constructive nonstandard analysis, Bulletin of Symbolic Logic, 3(1998), 233-272.

[14] E.A. Palyutin, Categorical Horn classes I, Algebra and Logic 19(1980), 377 - 400 (translation from Algebra i Logika); doi: 10.1007/BF01669610.

[15] F. Richman, Intuitionistic notions of boundedness in $\mathbb{N}$, Mathematical Logic Quarterly 55(2009), 31-36; doi: 10.1002/malq.200710072.

[16] C. Schmieden and D. Laugwitz, Eine Erweiterung der Infinitesimalrechnung, Mathematische Zeitschrift. 69(1958), 1-39; doi: 10.1007/BF01187391.

[17] P. Schuster, A constructive look at generalized Cauchy reals, Mathematical Logic Quarterly 46(1)(2000), 125-134; doi: 10.1002/(SICI)1521-3870(200001)46:1<125::AIDMALQ125>3.0.CO;2-V .

[18] A.S. Troelstra and D. van Dalen, Constructivism in Mathematics, vol. I and II, NorthHolland 1988.

Department of Mathematics, Stockholm University, SE-106 91 Stockholm, Sweden

palmgren@math.su.se

http://www.math.su.se/ palmgren

Received: 20 January 2011 Revised: 30 December 2011 